\title{
Fractional order heat conduction and thermoelastic response of a thermally sensitive rectangular parallelopiped
}

\author{
V. B. Srinivas ${ }^{1}$, V. R. Manthena ${ }^{2 *}$, Jaya Bikram ${ }^{1}$, G. D. Kedar ${ }^{1}$ \\ ${ }^{1}$ Department of Mathematics, RTM Nagpur University, Nagpur, INDIA. \\ ${ }^{2}$ Department of Mathematics, P. J. L. College of Engineering, Nagpur, INDIA. \\ *Corresponding Author Email: vkmanthena@gmail.com
}

Received 29 December 2020, Revised 13 February 2021, Accepted 14 February 2021

\begin{abstract}
In the present paper, the problem of finite dimensional rectangular parallelepiped in isotropic thermoelastic medium with convective type heating is considered. The heat conduction equation (HCE) of the region is described by time $\mathrm{HC}$ of fractional order with Caputo derivative form. The non-linear form of heat conduction equation is converted to linear form with Kirchhoff's transformation. Integral transform technique is used to deal with the spatial variables and Laplace transform technique is used to deal with Caputo type time fractional derivative. Inverse Laplace transform and inverse finite Fourier transform are employed to expose the solution in the transformed domain. Numerical results are obtained for temperature distribution, deflection, stress resultants and thermal stress distribution for different values of time fractional order parameter. These results are presented graphically and discussed for various values of time fractional parameters. The obtained results show significant influence of the time fractional order derivative on the temperature as well as stress distribution. Thermosensitivity plays a vital role in the analysis of any real thermoelastic problems and one should consider their effect while dealing with materials in high temperature environment.
\end{abstract}

Keywords: Fractional order; Heat conduction; Deflection; Stress resultants; Thermal stresses

\section{Introduction}

In the classical Fourier law, the heat conduction represents the relation between heat flow and change in temperature of a solid material. In the field of thermoelasticity the heat diffusion process in heterogeneous and non-regular materials like amorphous, glassy, dielectric, polymers, etc; the law of mechanics are not applicable and hence it becomes necessary to adopt the heat conduction equation with fractional order. The fractional calculus is the generalization of the derivative and integration of noninteger order. Many researchers worked in developing the theory of thermoelasticity in the field of science, where fractional calculus is applied and successful results have been obtained.

Caputo et al. [1-2] introduced time fractional derivative to stress-strain relation to obtain the analytic solution for a linear dissipative mechanism over large solution of fractional diffusion equation based on Riemann-Liouville fractional derivatives in terms of $\mathrm{H}$-functions and found that it admits a probabilistic frequency ranges and discussed various applications. The non-local theory of thermodynamics with constitutive equations is established for non-local thermoelastic solids by Eringen [3]. Noda [4] discussed thermal stresses in materials dependent on temperature. The theory of fractional calculus and its application is investigated by many researchers [5-8]. Heat conduction problems and their thermoelastic effect in thermosensitive bodies was discussed by [910]. Luchko and Gorenflo [11] introduced the operational method to obtain an exact solution of fractional differential equation of initial value problem. The proposed solutions are represented in Mittag-Leffler function. Gorenflo et al. [12] investigated a mapping of linear operator for various fractional parameters and also analyzed the exact solution for Green's function of the Cauchy problem. Hilfer [13] obtained the exact

interpretation in contrast with fractional diffusion based on fractional integrals. Gorenflo et al. [14-15] used Laplace transform for evaluating the linear 
operators of fractional integration and fractional differentiation. The results for fractional time order 0.5 , shows slow diffusion and for order 1.5 , exhibit mixed diffusion-wave behaviour as per [16].

Povstenko et al. [17-24] also solved the problem for time fractional heat diffusion equation with different approach. Deshmukh et al. [25] obtained thermal bending moments in a simply supported plate. Chain rule for fractional derivatives was discussed by Tarasov [26]. Manthena et al. [27] studied thermal stresses in a functionally graded (FG) plate. Popovych and Kalynyak [28] designed a mathematical model for thermally sensitive cylinders. Tripathi et al. [29] analyzed the fractional order thermoelastic problem with finite wave speeds. The thermoelastic displacement, stress and temperature are investigated in the thick circular plate of finite thickness and infinite extent, upper and lower surfaces are traction free. The thermal shock problem of an elastic half space in a fractional thermoelasticity is solved by [30]. Laplace transform and Hankel transform are applied to evaluate an initial-boundary value problem of fractional heat conduction. Warbhe et al. [31] analyzed fractional heat conduction and thermal deflection in a circular disk. The effect of thermosensitivity on a FG plate was studied by Manthena et al. [32-33].

Sherief and Raslan [34] proposed Caputo Fabrizio fractional differential operator to deal with theory of fractional thermoelasticity based on infinite elastic space subjected to continuous line source of heat. Zullo and Sciubba [35] shown that a system in an initial non-equilibrium state relaxes to equilibrium releasing (or absorbing) an additional amount of exergy, called non-equilibrium exergy, which is fundamentally different from Gibbs' Available Energy and depends on both the initial state and the imposed boundary conditions. Lazzaretto and Toffolo [36] developed a practical tool that is based on a new methodology, named SYNTHSEP, to generate new energy system configurations. Varghese et al. [37] determined the thermoelastic stresses in a thin elliptical plate made up of non-simple elastic material subjected to point impulsive time-dependent source of heat. Rajabi et al. [38] examined forced vibration behaviors for nonlocal strain gradient nanobeams with surface effects subjected to a moving harmonic load. Arani et al. [39] discussed static and dynamic response of nanoplate resting on an orthotropic visco-Pasternak foundation based on Eringen's nonlocal theory. Povstenko and Kyrylych [40] investigated a problem for an infinite solid containing penny-shaped crack and oscillation phenomena. Bhaskar et al. [7] analyzed the problem of linear uncoupled thermoelasticity and obtained the results for orthotropic and anisotropic composite laminates. Evaluated results are used to with its surface loaded at prescribed heat flux. Peng et al. [41] investigated the dynamic response of an infinite thermoelastic medium with a spherical cavity with fractional order derivative. Sherief and Hussein [42] analyzed two-dimensional axisymmetric problems in context with fractional order thermoelasticity. Köbler [43] shown that the temperature dependence of the heat capacity of the magnetic solids can completely be described by a sequence of universal power functions of temperature. Thakare et al. [44] studied thermoelastic problem of nonhomogeneous thick hollow cylinder within the context of fractional order derivative. NiezgodaŻelasko [45] studied entropy generation rate minimization during the flow of ethanol-based ice slurry through straight pipes with a circular and rectangular cross-section. Çoban and Kavas [46] solved Peng-Robinson cubic equation of states (EoS) for dry air. Mohammadi and Rahmani [47] investigated buckling analysis of a sandwich cylindrical shell with functionally graded face sheets and homogenous core. Molla-Alipour [48] developed a unified formulation for free vibration analysis of the bidirectional functionally graded conical and cylindrical shells and annular plates on elastic foundations. Sarvandi et al. [49] investigated the critical torsional load of a composite tube made of Polyvinylidene fluoride reinforced with double-walled boron nitride nanotubes. Shekarzadeh et al. [50] studied the free vibration analysis of a multi-layer rectangular plate with two magneto-rheological (MR) fluid layers and a flexible core. Pawar et al. [51] discussed the radially varying transient temperature distribution in a multilayer composite hollow sphere subjected to the time independent volumetric generation of heat in each layer. The theory and applications of thermoelasticity and fractional calculus was explained by [52-56].

In the present fractional thermoelastic problem, we considered linear integral and differential equations of fractional order under processes of some physical interest. The solutions are obtained for these problems are represented in Mittag-Leffler function form. Mainardi et al. [14-15] reviewed such problems and the governed equation of fractional order in which the solution is expressed in the form of Mittag-Leffler function. The properties of Mittag-Leffler function were discussed and the solution in Mittag-Leffler form including Abel integral equations of the second kind are obtained over generalized relaxation

check the accuracy of classical lamination theory based on Kirchhoff's hypothesis. It is concluded from a careful review of literature that the study of 
uncoupled problem in finite field is needed in fractional theory of thermoelasticity.

In the present investigation, we considered an isotropic thermoelastic rectangular parallelopiped in finite domain with time dependent fractional order derivative. The fractional heat conduction is subjected to convective heating along $x$-direction, while the other sides are thermally insulated. Integral transform technique is applied to find the expression for temperature distribution. The obtained solution in transformed domain is expressed in terms of MittagLeffler function. The effects of time fractional order parameter $r$ on temperature, deflection and thermal stresses are analyzed graphically. In this work, we have developed a mathematical model to study thermoelastic response of a rectangular parallelopiped using time fractional heat conduction equation. In the literature, we rarely found such kind of problems and hence it is innovative addition to the field of thermoelasticity.

\section{Formulation and solution of the heat conduction equation}

Consider a rectangular parallelopiped occupying the space defined as $0 \leq x \leq a, 0 \leq y \leq b, 0 \leq z \leq c$. A mathematical model is prepared for non-local Caputo type time fractional heat conduction equation (FHCE) of order $r$.

The definition of Caputo type fractional derivative is given by [1-2]

$$
\frac{\partial^{r} F(t)}{\partial t^{r}}=\frac{1}{\Gamma(h-r)} \int_{0}^{t}(t-\tau)^{h-r-1} \frac{d^{h} F(\tau)}{d \tau^{h}} d \tau, \quad h-1<r<h
$$

To find the Laplace transform, the Caputo derivative requires the initial values of the function $F(t)$ and its integral derivatives of the order $k=1,2,3, \ldots . . . h-1$.

$L\left[\frac{\partial^{r} F(t)}{\partial t^{r}}\right]=s^{r} \bar{F}(s)-\sum_{k=0}^{h-1} F^{(k)}\left(0^{+}\right) s^{r-1-k}, \quad h-1<r<h$

Here $s$ is the Laplace transform parameter.

The governing FHCE of a simply supported rectangular parallelopiped satisfies the differential equation $[46,47]$

$$
\begin{aligned}
& \frac{\partial}{\partial x}\left(k(T) \frac{\partial T}{\partial x}\right)+\frac{\partial}{\partial y}\left(k(T) \frac{\partial T}{\partial y}\right)+\frac{\partial}{\partial z}\left(k(T) \frac{\partial T}{\partial z}\right) \\
& =\rho[C(T)]^{r} \frac{\partial^{r} T}{\partial t^{r}}
\end{aligned}
$$

modulus and coefficient of linear thermal expansion, $\varpi_{1}, \varpi_{2}$ are the frequency. with boundary conditions

$$
\begin{array}{ll}
k(T) \frac{\partial T}{\partial x}-\varepsilon_{1} T=0, & \text { at } x=0 \\
k(T) \frac{\partial T}{\partial x}+\varepsilon_{2} T=f(y, z, t), & \text { at } x=a \\
\frac{\partial T}{\partial y}=0, & \text { at } y=0, b \\
\frac{\partial T}{\partial z}=0, & \text { at } z=0, c
\end{array}
$$

and initial conditions

$$
\begin{array}{lll}
T=0, & \text { at } t=0, & 0<r \leq 2 \\
\frac{\partial T}{\partial t}=0, & \text { at } t=0, & 1<r \leq 2
\end{array}
$$

where $k(T)$ and $C(T)$ are respectively, the temperature dependent thermal conductivity and specific heat capacity of the plate, $\rho$ is the density, and $\varepsilon_{1}, \varepsilon_{2}$ are the heat transfer coefficients.

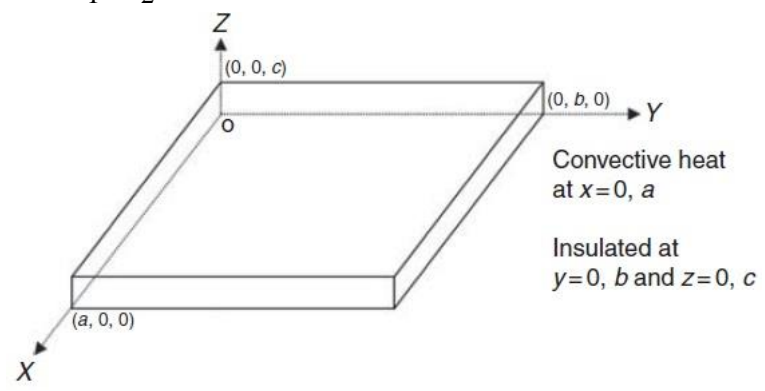

Fig. (1) Geometry of the problem

\section{Dimensionless Parameters}

$$
\begin{aligned}
& T *=\frac{T}{T_{0}},\left(x^{*}, y^{*}, z^{*}\right)=\frac{(x, y, z)}{a}, \\
& \left(a^{*}, b^{*}, c^{*}\right)=\frac{(a, b, c)}{a}, t *=\sqrt[r]{\kappa / a^{2}} t, E_{0} *=\frac{E_{0}}{E_{1}}, \\
& \alpha_{0} *=\frac{\alpha_{0}}{\alpha_{1}}, \rho^{*}=\frac{\rho}{\rho_{0}}, k_{0} *=\frac{k_{0}}{k_{1}}, \varpi_{1} *=\frac{\varpi_{1} a^{2}}{\kappa}, \\
& \varpi_{2} *=\frac{\varpi_{2} a^{2}}{\kappa} .
\end{aligned}
$$

where $T_{0}$ is the temperature of the surrounding environment, $\kappa=k_{0} /\left(C_{0} \rho_{0}\right)$, is the thermal diffusivity, $\rho_{0}$ is the reference value of density, $E_{0}, E_{1}, \alpha_{0}, \alpha_{1}$ are the reference values of Young's

The temperature dependent material properties $k(T), C(T)$, and heat flow $f(y, z, t)$ are taken as [9, $10,28]$ 


$$
\begin{aligned}
& k(T)=k_{0} k^{*}\left(T^{*}\right) \\
& {[C(T)]^{r}=C_{0}\left[C^{*}\left(T^{*}\right)\right]^{r}} \\
& f(y, z, t)=f_{0} f^{*}\left(y^{*}, z^{*}, t^{*}\right)
\end{aligned}
$$

where $k_{0}, k_{1}, C_{0}$ are the reference values of thermal conductivity, specific heat capacity having dimensions, $f_{0}$ is the strength of the heat flow having relevant dimensions, and $k^{*}\left(T^{*}\right), C^{*}\left(T^{*}\right)$ are the dimensionless quantities, which are functions that describe the dependence of these characteristics on dimensionless temperature, $f *\left(y^{*}, z^{*}, t^{*}\right)$ is the dimensionless function which describes the space distribution of the heat flow.

Using equations (6-7), equations (3-5) reduces to the following dimensionless form (ignoring asterisks for convenience).

$$
\begin{aligned}
& \frac{\partial}{\partial x}\left(k(T) \frac{\partial T}{\partial x}\right)+\frac{\partial}{\partial y}\left(k(T) \frac{\partial T}{\partial y}\right)+\frac{\partial}{\partial z}\left(k(T) \frac{\partial T}{\partial z}\right) \\
& =\rho[C(T)]^{r} \frac{\partial^{r} T}{\partial t^{r}}
\end{aligned}
$$

boundary conditions

$$
\begin{array}{ll}
k(T) \frac{\partial T}{\partial x}-B i_{1} T=0, & \text { at } x=0 \\
k(T) \frac{\partial T}{\partial x}+B i_{2} T=K i f(y, z, t), & \text { at } x=a \\
\frac{\partial T}{\partial y}=0, & \text { at } y=0, b \\
\frac{\partial T}{\partial z}=0, & \text { at } z=0, c
\end{array}
$$

initial conditions

$$
\begin{aligned}
& T=0, \quad \text { at } t=0, \quad 0<r \leq 2 \\
& \frac{\partial T}{\partial t}=0, \quad \text { at } t=0, \quad 1<r \leq 2
\end{aligned}
$$

where $K i=\left(f_{0} a\right) /\left(k_{0} T_{0}\right)$ is the dimensionless Kirpichev reference number, $B i_{j}=\left(\varepsilon_{j} a\right) /\left(k_{0}\right), j=1,2$, is the Biot criteria. Introducing the Kirchhoff's variable transformation $\Theta(T)=\int_{0}^{T} k(T) d T$

in equation (8) and taking into account that the material with simple thermal nonlinearity is considered, we obtain

$$
\frac{\partial^{2} \Theta}{\partial x^{2}}+\frac{\partial^{2} \Theta}{\partial y^{2}}+\frac{\partial^{2} \Theta}{\partial z^{2}}=\rho \frac{\partial^{r} \Theta}{\partial t^{r}}
$$

The boundary conditions (9) become

$$
\begin{array}{ll}
\frac{\partial \Theta}{\partial x}-B i_{1} \Theta=0, & \text { at } x=0 \\
\frac{\partial \Theta}{\partial x}+B i_{2} \Theta=K i f(y, z, t), & \text { at } x=a \\
\frac{\partial \Theta}{\partial y}=0, & \text { at } y=0, b \\
\frac{\partial \Theta}{\partial z}=0, & \text { at } z=0, c
\end{array}
$$

The initial conditions (10) become

$\Theta=0, \quad$ at $t=0, \quad 0<r \leq 2$

$\frac{\partial \Theta}{\partial t}=0, \quad$ at $t=0, \quad 1<r \leq 2$

For the sake of brevity, we take

$$
f(y, z, t)=\delta\left(y-y_{0}\right) \delta\left(z-z_{0}\right) \delta(t) .
$$

This represents an instantaneous heat flow at the point $\left(a, y_{0}, z_{0}\right), y_{0}, z_{0}$ being dimensionless constants.

To solve the heat conduction equation (12) subjected to convective boundary conditions defined in equation (13) over the space variable $x$ in $0 \leq x \leq a$, first we define the integral transform and its inversion formula for the temperature function $\Theta(x, y, z, t)$ as [47]

$$
\begin{aligned}
\left(\begin{array}{l}
\text { Integral } \\
\text { Transform }
\end{array}\right) & \bar{\Theta}\left(\eta_{m}, y, z, t\right) \\
= & \int_{x^{\prime}=0}^{a} R\left(\eta_{m}, x^{\prime}\right) \Theta\left(x^{\prime}, y, z, t\right) d x^{\prime} \\
\left(\begin{array}{l}
\text { Inversion } \\
\text { Formula }
\end{array}\right) & \Theta(x, y, z, t) \\
= & \sum_{m=1}^{\infty} R\left(\eta_{m}, x\right) \bar{\Theta}\left(\eta_{m}, y, z, t\right)
\end{aligned}
$$

Here $R\left(\eta_{m}, x\right)$ is the kernel of the transform given by

$$
R\left(\eta_{m}, x\right)=\Psi_{1}\left(\eta_{m} \cos \eta_{m} x+B i_{1} \sin \eta_{m} x\right)
$$

where

$$
\Psi_{1}=\left[\sqrt{2} / \sqrt{\left(\eta_{m}^{2}+B i_{1}^{2}\right)\left(a+\frac{B i_{2}}{\eta_{m}^{2}+B i_{2}^{2}}\right)+B i_{1}}\right]
$$

Here $\eta_{m}$ 's are the positive roots of the transcendental equation $\tan \eta_{m} a=\frac{\eta_{m}\left(B i_{1}+B i_{2}\right)}{\eta_{m}^{2}-B i_{1} B i_{2}}$

Implementing integral transform defined in equation (16), Finite Fourier cosine transform, and Laplace transform on equation (12), we arrive at 


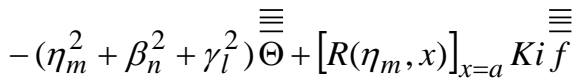

$$
\begin{aligned}
& -\left[R\left(\eta_{m}, x\right)\right]_{x=a} B i_{2} \overline{\overline{\bar{\Theta}}}+\left[R\left(\eta_{m}, x\right)\right]_{x=0} B i_{1} \equiv \\
& =\rho s r \overline{\bar{\Theta}}
\end{aligned}
$$

where

$$
\beta_{n}=n \pi y / b, \gamma_{l}=l \pi z / c
$$

and

$\cos (n \pi y / b), \cos (1 \pi z / c)$, being the kernels of finite Fourier cosine transform.

The value of the kernel $R\left(\eta_{m}, x\right)$ at $x=a$ and $x=0$ is obtained as follows

$$
\begin{aligned}
{\left[R\left(\eta_{m}, x\right)\right]_{x=a} } & =\Psi_{1}\left(\eta_{m} \cos \eta_{m} a+B i_{1} \sin \eta_{m} a\right) \\
& =P_{a}(\text { say }) \\
{\left[R\left(\eta_{m}, x\right)\right]_{x=0} } & =\Psi_{1} \eta_{m}=P_{0} \text { (say) }
\end{aligned}
$$

On simplification equation (19) leads to

$$
\overline{\bar{\Theta}}=\frac{P_{a} K i}{\rho} \frac{\overline{\overline{\bar{f}}}}{\left(s^{r}+\left(\left(\eta_{m}^{2}+\beta_{n}^{2}+\gamma_{l}^{2}+P_{a} B i_{2}-P_{0} B i_{1}\right) / \rho\right)\right)}
$$

Taking inverse Laplace transform, eqn. (21) becomes

$$
\begin{aligned}
& \overline{\overline{\bar{\Theta}}}=\frac{P_{a} K i}{\rho} \\
& \times \int_{s=0}^{\infty} \frac{\overline{\bar{f}} e^{-s t}}{\left(s^{r}+\left(\left(\eta_{m}^{2}+\beta_{n}^{2}+\gamma_{l}^{2}+P_{a} B i_{2}-P_{0} B i_{1}\right) / \rho\right)\right)} d s
\end{aligned}
$$

Applying inverse integral transform defined in equation (16) over the variable $x$, and inverse Fourier cosine transform over the variables $y, z$, equation (22) leads to

$$
\begin{aligned}
& \Theta=\frac{P_{a} K i}{\rho} \sum_{m=1}^{\infty}\left\{\left[\left[\overline{\bar{\Theta}}\left(\eta_{m}, \beta_{n}, z, t\right)\right]_{n=0} / b\right]\right. \\
& \left.+(2 / b) \sum_{n=1}^{\infty}\left\{\overline{\bar{\Theta}}\left(\eta_{m}, \beta_{n}, z, t\right) \times \cos (n \pi y / b)\right\}\right\} \\
& \times\left\{\left(\left(\eta_{m} \cos \eta_{m} x+B i_{1} \sin \eta_{m} x\right)\right)\right. \\
& \left.\times\left(\sin \eta_{m} a-\left(B i_{1} / \eta_{m}\right) \cos \eta_{m} a+\left(B i_{1} / \eta_{m}\right)\right)\right\} \\
& \text { where } \\
& \overline{\bar{\Theta}}\left(\eta_{m}, \beta_{n}, z, t\right)=\left[\left[\xi_{1}(t)\right]_{l=0} / c\right]+(2 / c) \\
& \quad \times \sum_{l=1}^{\infty} \xi_{1}(t) \times \cos (l \pi z / c) \\
& \xi_{1}(t)=E_{r, 1}\left(-\left(\left(\eta_{m}^{2}+\beta_{n}^{2}+\gamma_{l}^{2}+P_{a} B i_{2}-P_{0} B i_{1}\right) / \rho\right) t^{r}\right)
\end{aligned}
$$

is the Mittag-Leffler function and in summation form it is represented as

$$
\begin{aligned}
& E_{r, 1}\left(-\left(\left(\eta_{m}^{2}+\beta_{n}^{2}+\gamma_{l}^{2}+P_{a} B i_{2}-P_{0} B i_{1}\right) / \rho\right) t^{r}\right) \\
& =\sum_{j=0}^{\infty}(-1)^{j}\left(\left(\left(\eta_{m}^{2}+\beta_{n}^{2}+\gamma_{l}^{2}+P_{a} B i_{2}-P_{0} B i_{1}\right) / \rho\right)\right) t^{r j}
\end{aligned}
$$

Applying inverse Kirchhoff's variable transform on equation (23) [Refer Appendix A], the solution of temperature distribution is obtained as

$$
\begin{aligned}
& T(x, y, z, t) \cong \frac{P_{a} K i}{k_{0} \rho} \sum_{m=1}^{\infty}\left\{\left[\left[\overline{\bar{\Theta}}\left(\eta_{m}, \beta_{n}, z, t\right)\right]_{n=0} / b\right]\right. \\
& +(2 / b) \sum_{n=1}^{\infty}\left\{\left\{\left[\left[\xi_{1}(t)\right]_{l=0} / c\right]+(2 / c) \times \sum_{l=1}^{\infty} \xi_{1}(t)\right.\right. \\
& \times \cos (l \pi z / c)\} \times \cos (n \pi y / b)\}\} \times\left\{\left(\left(\eta_{m} \cos \eta_{m} x\right.\right.\right. \\
& \left.\left.+B i_{1} \sin \eta_{m} x\right)\right) \\
& \left.\times\left(\sin \eta_{m} a-\left(B i_{1} / \eta_{m}\right) \cos \eta_{m} a+\left(B i_{1} / \eta_{m}\right)\right)\right\}
\end{aligned}
$$

\section{Thermoelastic analysis}

We consider a simply supported thin rectangular parallelopiped subjected to thermal load. The fundamental equation and the corresponding boundary conditions in the Cartesian coordinate system are [46]

$$
\nabla^{4} w=\frac{-1}{(1-v(T)) D(T)} \nabla^{2} M_{T}
$$

where

$$
\nabla^{2}=\frac{\partial^{2}}{\partial x^{2}}+\frac{\partial^{2}}{\partial y^{2}}+\frac{\partial^{2}}{\partial z^{2}}, \quad D(T)=\frac{E(T) c^{3}}{12\left(1-v(T)^{2}\right)}
$$

with

$$
\begin{aligned}
& w=0, \quad \frac{\partial^{2} w}{\partial x^{2}}=\frac{-1}{(1-v(T)) D(T)} M_{T} \text { at } x=0, a \\
& w=0, \quad \frac{\partial^{2} w}{\partial y^{2}}=\frac{-1}{(1-v(T)) D(T)} M_{T} \text { at } y=0, b
\end{aligned}
$$

where $w$ is the deflection, $M_{T}$ is the thermally induced resultant moment, $D(T), E(T), v(T)$ are respectively, the temperature dependent bending rigidity, Young's modulus and Poisson's ratio.

Considering the equilibrium state in the in-plate directions of $x$ and $y$, the in-plate resultant forces are

$N_{x}=N_{y}=N_{x y}=0$

The stress resultants per unit length of the rectangular parallelopiped are given as 


$$
\begin{aligned}
& M_{x}=-D(T)\left(\frac{\partial^{2} w}{\partial x^{2}}+v(T) \frac{\partial^{2} w}{\partial y^{2}}\right)-\frac{1}{1-v(T)} M_{T} \\
& M_{y}=-D(T)\left(v(T) \frac{\partial^{2} w}{\partial x^{2}}+\frac{\partial^{2} w}{\partial y^{2}}\right)-\frac{1}{1-v(T)} M_{T} \\
& M_{x y}=(1-v(T)) D(T) \frac{\partial^{2} w}{\partial x \partial y}
\end{aligned}
$$

The stress components in terms of stress resultants are

$$
\begin{aligned}
\sigma_{x x}= & \frac{1}{c} N_{x}+\frac{12 z}{c^{3}} M_{x}+\frac{1}{1-v(T)} \\
& \times\left(\frac{1}{c} N_{T}+\frac{12 z}{c^{3}} M_{T}-\alpha(T) E(T) T\right) \\
\sigma_{y y}= & \frac{1}{c} N_{y}+\frac{12 z}{c^{3}} M_{y}+\frac{1}{1-v(T)} \\
& \times\left(\frac{1}{c} N_{T}+\frac{12 z}{c^{3}} M_{T}-\alpha(T) E(T) T\right) \\
\sigma_{x y}= & \frac{1}{c} N_{x y}-\frac{12 z}{c^{3}} M_{x y} \\
M_{T}= & \int_{0}^{c} \alpha(T) E(T) T z d z, N_{T}=\int_{0}^{c} \alpha(T) E(T) T d z
\end{aligned}
$$

Here $\alpha(T)$ is the temperature dependent coefficient of linear thermal expansion.

To find the moments $M_{T}$ and $N_{T}$, we take

$$
\begin{aligned}
& E(T)=E_{0} \exp \left(\varpi_{1} T\right), \alpha(T)=\alpha_{0} \exp \left(\varpi_{2} T\right), \\
& v(T)=v_{0} \exp \left(\varpi_{2} T\right), \varpi_{1} \leq 0, \varpi_{2} \geq 0
\end{aligned}
$$

Using equations (34) and (26) in equation (33), we obtain

$$
\begin{aligned}
& M_{T}=\sum_{m=1}^{\infty}\left\{( 2 / b ) \sum _ { n = 1 } ^ { \infty } \left\{L_{1} \times\left\{\left[\left[\xi_{1}(t)\right]_{l=0} / c\right]\right.\right.\right. \\
& \left.\left.\left.+(2 / c) \times \sum_{l=1}^{\infty} \xi_{1}(t)\right\} \times \cos (n \pi y / b)\right\}\right\} \\
& \times\left\{\left(\left(\eta_{m} \cos \eta_{m} x+B i_{1} \sin \eta_{m} x\right)\right)\right. \\
& \left.\times\left(\sin \eta_{m} a-\left(B i_{1} / \eta_{m}\right) \cos \eta_{m} a+\left(B i_{1} / \eta_{m}\right)\right)\right\} \\
& N_{T}=\sum_{m=1}^{\infty}\left\{( 2 / b ) \sum _ { n = 1 } ^ { \infty } \left\{L_{2} \times\left\{\left[\left[\xi_{1}(t)\right]_{l=0} / c\right]\right.\right.\right. \\
& \left.\left.\left.+(2 / c) \times \sum_{l=1}^{\infty} \xi_{1}(t)\right\} \times \cos (n \pi y / b)\right\}\right\} \\
& \times\left\{\left(\left(\eta_{m} \cos \eta_{m} x+B i_{1} \sin \eta_{m} x\right)\right)\right. \\
& \left.\times\left(\sin \eta_{m} a-\left(B i_{1} / \eta_{m}\right) \cos \eta_{m} a+\left(B i_{1} / \eta_{m}\right)\right)\right\}
\end{aligned}
$$

temperature takes non-zero value at both the ends. The speed of propagation of thermal signals is directly where

$$
\begin{aligned}
& L_{1}=\left(P_{a} K i \alpha_{0} E_{0} / 9 b^{3} c k_{0} \rho \pi^{2} l^{2}\right)\left(9 b^{2} c^{2}\right. \\
& \left(-4+c \pi^{2} l^{2}\right)+4(-112+9 c(-3(1+4 c) \\
& \left.+\left(6+c^{2}\right) \pi^{2} l^{2}\right) \varpi_{1} \varpi_{2}+18 b c(-1-8 c \\
& \left.+\left(2+c^{2}\right) \pi^{2} l^{2}\right)\left(\varpi_{1}+\varpi_{2}\right)+36\left(b^{2} c^{2}\right. \\
& +12\left(1+c^{2}\right) \varpi_{1} \varpi_{2}+4 b c^{2}\left(\varpi_{1}+\varpi_{2}\right) \cos (l \pi) \\
& +18 c\left(6 \varpi_{1} \varpi_{2}+b\left(\varpi_{1}+\varpi_{2}\right)\right) \cos (2 l \pi) \\
& +18 \varpi_{1} \varpi_{2} \cos (3 l \pi) \\
& L_{2}=\left(2 P_{a} K i c \alpha_{0} E_{0} / 3 b^{3} c^{2} k_{0} \rho \pi l\right)(3 c \pi l \\
& \left.\left(b^{2} c^{2}+4\left(6+c^{2}\right) \varpi_{1} \varpi_{2}+2 b\left(2+c^{2}\right)\left(\varpi_{1}+\varpi_{2}\right)\right)\right) .
\end{aligned}
$$

Using equation (35), the deflection $w$ from equation (27), the stress resultants and the corresponding stress components from equations (31) and (32), using deflection and thermally induced resultant moments, are obtained using Mathematica Software.

\section{Numerical results and discussion}

For numerical computations, we consider a mathematical model with Copper material having the following thermo-mechanical parameters.

$$
\begin{aligned}
& T_{0}=320 \mathrm{~K}, \quad a=4 \mathrm{~m}, \mathrm{~b}=2 \mathrm{~m}, \mathrm{c}=1 \mathrm{~m}, \\
& k_{0}=386 \mathrm{~W} / \mathrm{mK}, k_{1}=370 \mathrm{~W} / \mathrm{mK}, \\
& \rho=8954 \mathrm{~kg} / \mathrm{m}^{3}, \rho_{0}=8952 \mathrm{~kg} / \mathrm{m}^{3}, \\
& C_{0}=388 \mathrm{~J} / \mathrm{kgK}, \quad \kappa=11.1 \times 10^{-5} \mathrm{~m}^{2} / \mathrm{sec}, \\
& E_{0}=133 \times 10^{9} \mathrm{~kg} / \mathrm{msec}^{2}, \\
& E_{1}=121 \times 10^{9} \mathrm{~kg} / \mathrm{msec}^{2}, \quad \alpha_{0}=17.9 \times 10^{-6} / \mathrm{K}, \\
& \alpha_{1}=16.8 \times 10^{-6} / \mathrm{K}, v_{0}=0.35, t=2 \mathrm{sec} .
\end{aligned}
$$

The following figures ( 2 to 9 ) show the variations of dimensionless temperature, deflection, stress resultants and thermal stresses. The figures ( 3 to 9 ) on the left are plotted for the homogeneous case (i.e. taking $\varpi_{1}=\varpi_{2}=0$, so that the material properties become independent of temperature), whereas that on the right are plotted for the nonhomogeneous case (i.e. taking $\varpi_{1}, \varpi_{2} \neq 0$, so that the material properties become dependent of temperature). All the graphs are plotted for fractional order parameter $r=0.5,1,1.5,2$ depicting weak, normal and strong conductivity.

Fig. (2) represents the temperature distribution along $x$-axis. It is seen that, the temperature follows a uniform pattern for different values of fractional order parameter with respect to $x$-coordinate. The

proportional to the values of fractional order parameter $r$. The magnitude of temperature increases from the 
outer end and becomes peak in the middle region and decreases towards the origin.

Fig. (3) represents the thermal deflection along $x$ axis. It is observed that, in both the homogeneous and nonhomogeneous cases, the deflection is positive at both the ends while negative in the middle region.

Figs. (4-6) represent the variation of dimensionless stress resultants along $x$-axis. The stress resultants $M_{x}, M_{y}$ are tensile in the middle region, while compressive at the both ends, while the stress resultant $M_{x y}$ exhibits a different nature. It is compressive in nature in the range $0<x<0.5$, while tensile in $0.5<x<1$.
Figs. (7-9) represent the variation of dimensionless stresses along $x$-axis. In the homogeneous case, the stress components $\sigma_{x x}, \sigma_{y y}$ are compressive at the outer end and tensile in the middle region. The stress component $\sigma_{x y}$ is tensile in $0<x<0.5$, while compressive in the remaining region. In the nonhomogeneous case, the stress components $\sigma_{x x}, \sigma_{y y}$ are tensile in $0<x<0.3$, while compressive in the remaining region, while $\sigma_{x y}$ assumes nearly zero value in the region is $0<x<0.5$ and becomes compressive till $x=0.95$ and suddenly rises at the outer end.

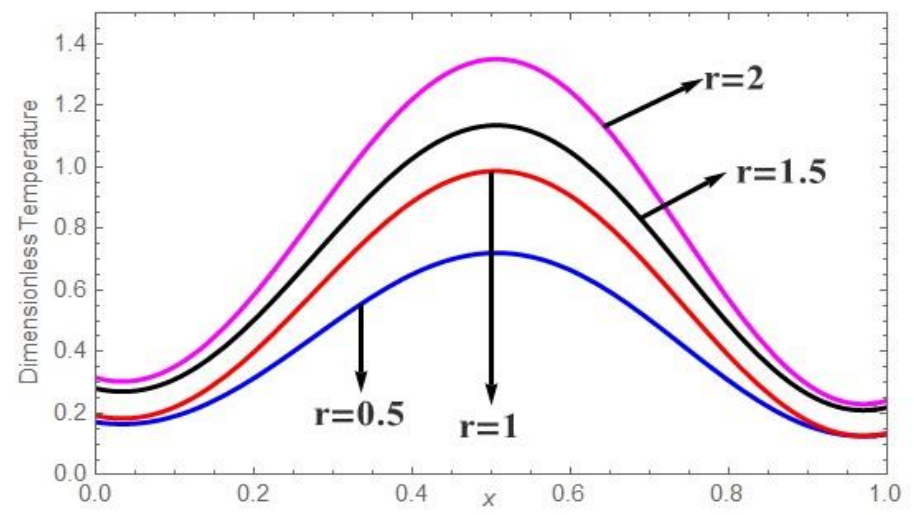

Fig. (2) Plot of dimensionless temperature along $x$-axis
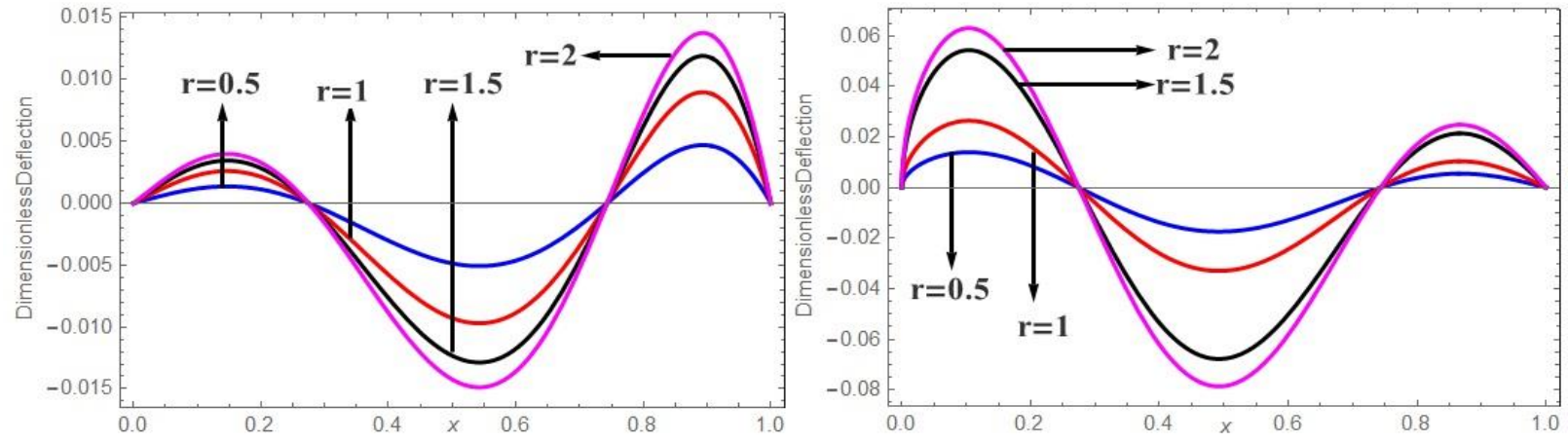

Fig. (3) Plot of dimensionless deflection along $x$-axis 

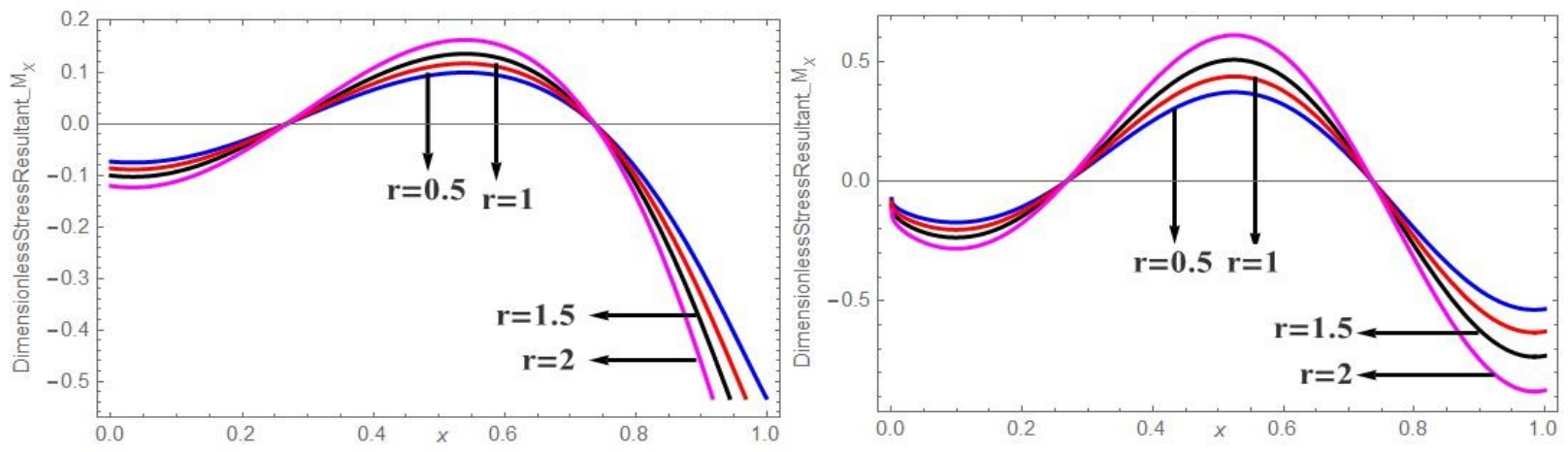

Fig. (4) Plot of dimensionless stress resultant $M_{x}$ along $x$-axis
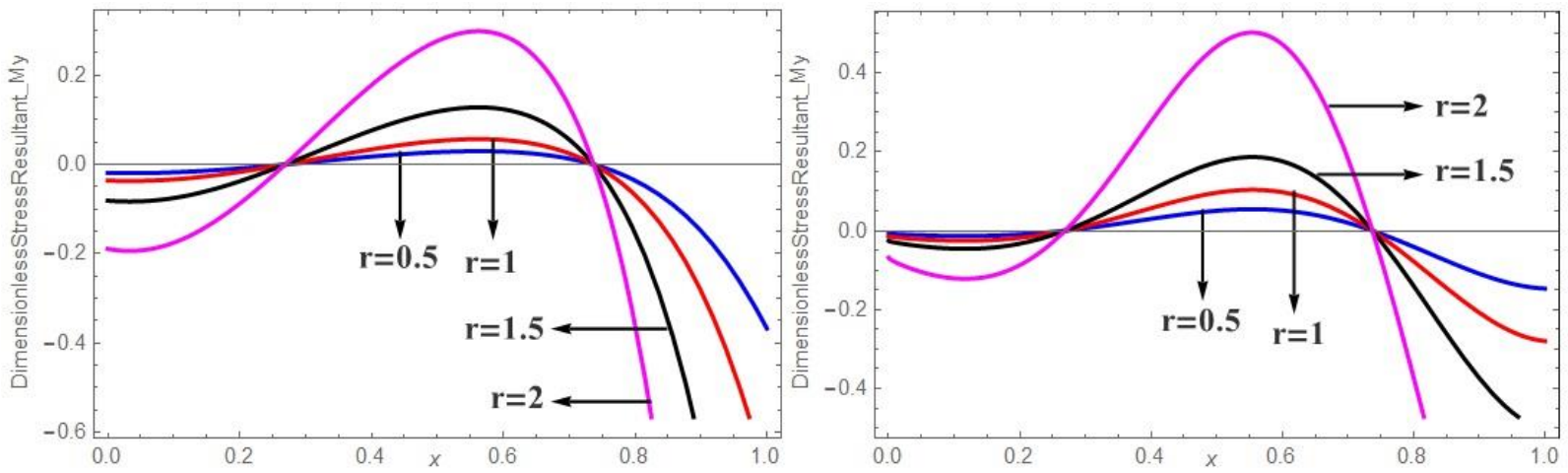

Fig. (5) Plot of dimensionless stress resultant $M_{y}$ along $x$-axis
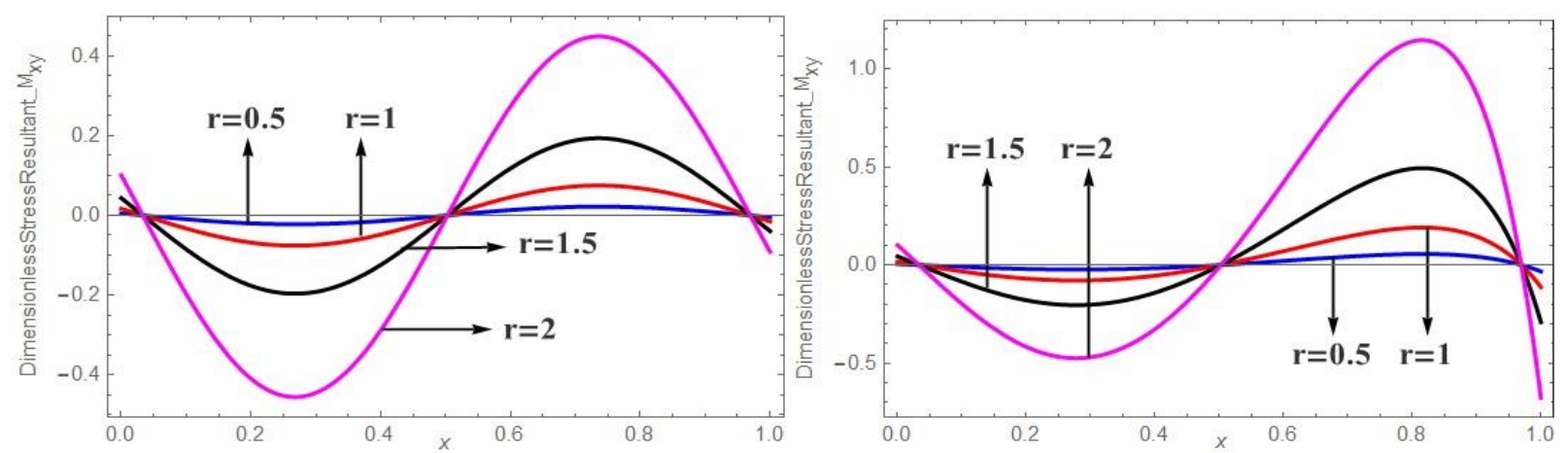

Fig. (6) Plot of dimensionless stress resultant $M_{x y}$ along $x$-axis
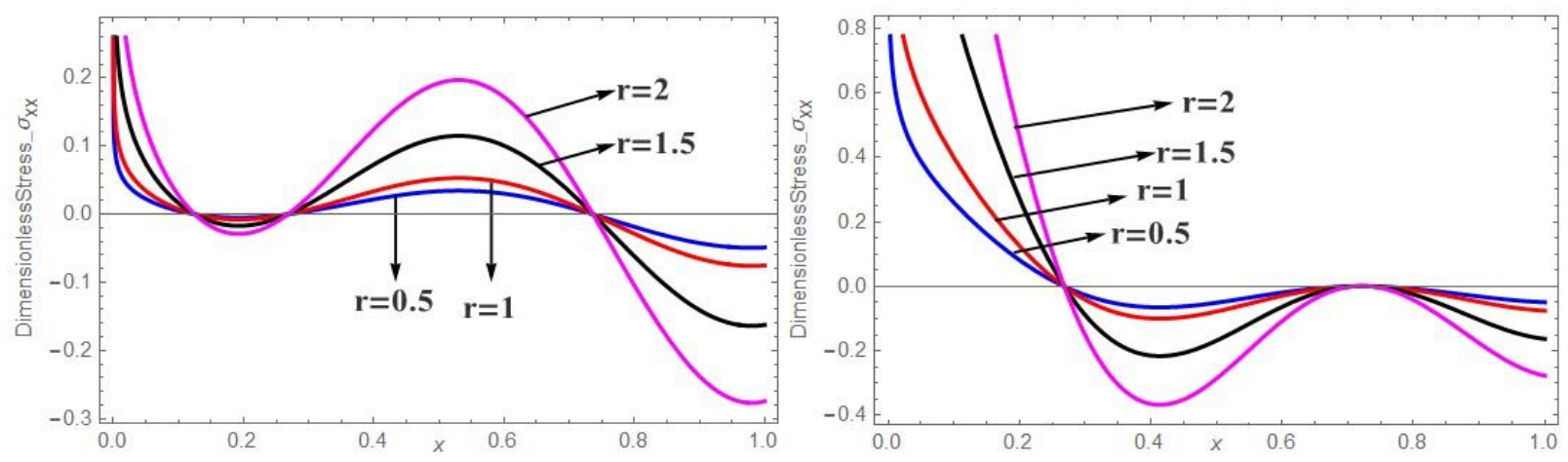

Fig. (7) Plot of dimensionless stress $\sigma_{x x}$ along $x$-axis 

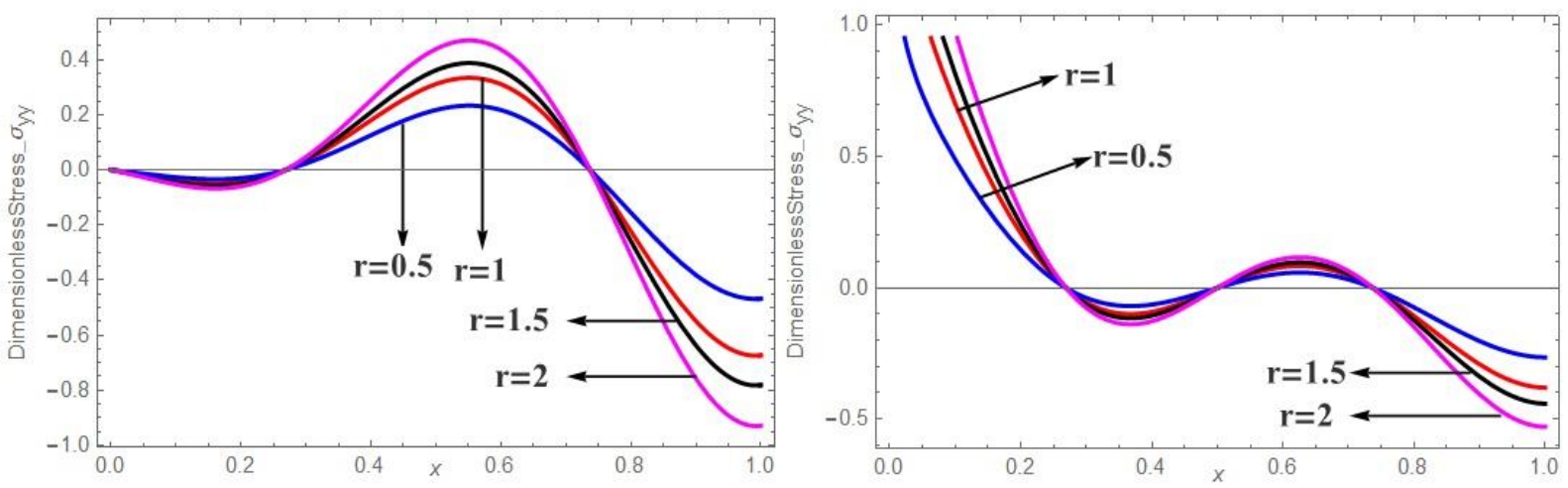

Fig. (8) Plot of dimensionless stress $\sigma_{y y}$ along $x$-axis
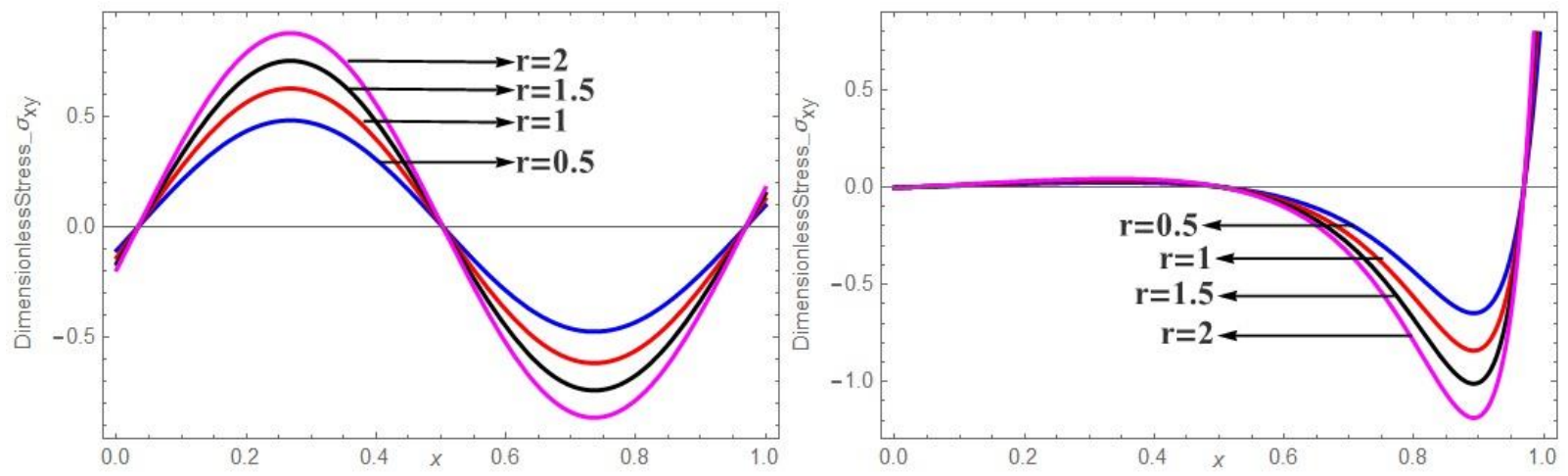

Fig. (9) Plot of dimensionless stress $\sigma_{x y}$ along $x$-axis

\section{Validation of the results}

In this paper, an analytical mathematical model has been prepared for a thermally sensitive rectangular parallelopiped and its temperature and stress profile is studied. As a limiting case, if we take $g(x, y, z, t)=0, r=1$, the results agree with [27].

\section{Conclusion}

In this paper, we have obtained solution of time-fractional heat conduction equation with the nonlocal type Caputo time-fractional derivative for a thermally sensitive rectangular parallelopiped. The obtained solutions reduce to the solutions of classical HCE for $r=1$. For $0<r<1$, the considered equation interpolates the elliptic Helmholtz equation $(r=0)$ and parabolic HCE. For $1<r<2$, the HCE interpolates the parabolic HCE and the hyperbolic wave equation, and the proposed theory of thermal stresses interpolates the classical thermoelasticity $(r=1)$ and that without energy dissipation $(r=2)$. The numerical results demonstrate significant influence of the fractional order of time derivative on the temperature as well as stress distribution. The fractional order parameter $0<r<1$ corresponds to weak conductivity, while $1<r<2$ corresponds to strong conductivity and $r=1$ corresponds to normal conductivity. The fractional order theory predicts a lagging response to physical stimuli, as observed in nature. The temperature-dependent material properties significantly affect the variations of the considered variables. The results indicate that, one needs to consider the effect of temperature dependent material properties in the analysis of any real thermoelastic problem. The presented results may be useful for designing of new materials, researchers in material sciences, researchers in high-temperature physics, and those working to further develop the theory of thermoelasticity with fractional calculus. 


\section{References}

[1] M. Caputo, Linear models of dissipation whose Q is almost frequency independent, Part II, Geophysical Journal International, 13, 529-539, 1967.

[2] M. Caputo, F. Mainardi, Linear models of dissipation in anelastic solids, Riv. Nuovo Cimento, 1,161-198, 1971.

[3] A. C. Eringen, Theory of nonlocal thermoelasticity, Int. J. Eng. Sci., 12, 1063-1077, 1974.

[4] N. Noda, Thermal stresses in materials with temperature dependent properties, Thermal Stresses I, North Holland, Amsterdam, 391-483, 1986.

[5] S. G. Samko, A. A. Kilbas, O. I. Marichev, Fractional Integrals and Derivatives: Theory and Applications, Gordon and Breach, Newark, 1993.

[6] K. S. Miller, B. Ross, An Introduction to the Fractional Calculus and Fractional Differential Equations, Wiley, New York, 1993.

[7] K. Bhaskar, T. K. Varadan, J. Ali, Thermoelastic solutions for orthotropic and anisotropic composite laminates, Composites Part B: Engineering, 27, 415-420, 1996.

[8] I. Podlubny, Fractional Differential Equations, Academic Press, New York, 1999.

[9] V. S. Popovich, B. N. Fedai, The axisymmetric problem of thermoelasticity of a multilayer thermosensitive tube, Journal of Mathematical Sciences, 86, 2605-2610, 1997.

[10] V. S. Popovych, I. M. Makhorkin, On the solution of heat conduction problems for Thermosensitive Bodies, Journal of Mathematical Sciences, 88, 352-359, 1998.

[11] Y. Luchko, R. Gorenflo, An operational method for solving fractional differential equations with the Caputo derivatives, Acta Mathematica Vietnamica, 24, 207-233, 1999.

[12] R. Gorenflo, A. Iskenderov, Y. Luchko, Mapping between solutions of fractional diffusion-wave equations, Fractional Calculus Appl. Anal., 3, 75-86, 2000.

[13] R. Hilfer, Fractional diffusion based on Riemann-Liouville fractional Derivatives, Journal of Physical Chemistry B, 104, 3914-3917, 2000.

[14] F. Mainardi, R. Gorenflo, On MittagLeffler-type functions in fractional evolution processes, J. Comput. Appl. Math., 118, 283-299, 2000.
[15] R. Gorenflo, F. Mainardi, D. Moretti, P. Paradisi, Time fractional diffusion: a discrete random walk approach, Nonlin. Dynamics, 29, 129-143, 2002.

[16] O.P. Agrawal, Solution for a fractional diffusion-wave equation defined in a bounded domain, Nonlinear Dynam., 29, 145-155, 2002.

[17] Y. Povstenko, Fractional heat conduction equation and associated thermal stresses, J. Thermal Stresses, 28, 83-102, 2005.

[18] Y. Povstenko, Stresses exerted by a source of diffusion in a case of a non-parabolic diffusion equation, International Journal of Engineering Science, 43, 977-991, 2005.

[19] Y. Povstenko, Two-dimensional axisymmetric stresses exerted by instantaneous pulses and sources of diffusion in an infinite space in a case of time-fractional diffusion equation, International Journal of Solids and Structures, 44, 2324-2348, 2007.

[20] Y. Povstenko, Fundamental solutions to central symmetric problems for fractional heat conduction equation and associated thermal stresses, Journal of Thermal Stresses, 31, 127148, 2007.

[21] Y. Povstenko, Fundamental solutions to three-dimensional diffusion-wave equation and associated diffusive stresses, Chaos, Solitons \& Fractals, 36, 961-972, 2008.

[22] Y. Povstenko, Signaling problem for time-fractional diffusion-wave equation in a halfplane, Fract. Calc. Appl. Anal., 11, 329-352, 2008.

[23] Y. Povstenko, Time-fractional radial heat conduction in a cylinder and associated thermal stresses, Archive of Applied Mechanics, 82, 345-362, 2012.

[24] Y. Povstenko, Fractional thermoelasticity, New York: Springer, 219, 2015. [25] K. C. Deshmukh, M. V. Khandait, R. Kumar, Thermal stresses in a simply supported plate with thermal bending moments with heat sources, Material Physics and Mechanics, 21, 135-146, 2014.

[26] V. E. Tarasov, On chain rule for fractional derivatives, Communications in Nonlinear Science and Numerical Simulation, 30, 1-4, 2016.

[27] V. R. Manthena, N. K. Lamba, G. D. Kedar, K. C. Deshmukh, Effects of stress resultants on thermal stresses in a functionally graded rectangular plate due to temperature 
dependent material properties, International Journal of Thermodynamics, 19, 235-242, 2016.

[28] V. S. Popovych, B. M. Kalynyak, Mathematical modeling and methods for the determination of the static thermoelastic state of multilayer thermally sensitive cylinders, Journal of Mathematical Sciences, 215, 218-242, 2016.

[29] J. J. Tripathi, G. D. Kedar, K. C. Deshmukh, Dynamic problem of fractional order thermoelasticity for a thick circular plate with finite wave speeds, Journal of Thermal Stresses, 39, 220-230, 2016.

[30] X. Y. Zhang, Z. T. Chen, X. F. Li, Thermal shock fracture of an elastic half-space with a subsurface penny-shaped crack via fractional thermoelasticity, Acta Mechanica, 229, 4875-4893, 2018.

[31] S. D. Warbhe, J. J. Tripathi, K. C. Deshmukh, J. Verma, Fractional heat conduction in a thin hollow circular disk and associated thermal deflection, Journal of Thermal Stresses, 41, 262-270, 2018.

[32] V. R. Manthena, G. D. Kedar, K. C. Deshmukh, Thermal stress analysis of a thermosensitive functionally graded rectangular plate due to thermally induced resultant moments, Multidiscipline Modeling in Materials and Structures, 14, 857-873, 2018.

[33] V. R. Manthena, G. D. Kedar, "On thermoelastic problem of a thermosensitive functionally graded rectangular plate with instantaneous point heat source", Journal of Thermal Stresses, 42, 849-862, 2019.

[34] H. H., Sherief, W. E. Raslan, Fundamental solution for a line source of heat in the fractional order theory of thermoelasticity using the new Caputo definition, Journal of Thermal Stresses, 42, 18-28, 2019.

[35] F. Zullo, E. Sciubba, On the quantification of non-equilibrium exergy for thermodynamic systems evolving according to Cattaneo's equation, International Journal of Thermodynamics, 22, 19-24, 2019.

[36] A. Lazzaretto, A. Toffolo, A practical tool to generate complex energy system configurations based on the synthsep methodology, International Journal of Thermodynamics, 22, 45-53, 2019.

[37] V. Varghese, P. Bhad, A. Mahakalkar, Temperature and stress distribution in a nonsimple elastic elliptical plate due to point impulsive heat sources, International Journal of Thermodynamics, 22, 65-72, 2019.

[38] K. Rajabi, Sh. H. Hashemi, A. R. Nezamabadi, Size-Dependent forced vibration analysis of three nonlocal strain gradient beam models with surface effects subjected to moving harmonic loads, Journal of Solid Mechanics, 11, 39-59, 2019.

[39] A. G. Arani, M. H. Jalaei, S. Niknejad, A. A. G. Arani, Size-Dependent analysis of orthotropic mindlin nanoplate on orthotropic visco-pasternak substrate with consideration of structural damping, Journal of Solid Mechanics, 11, 236-253, 2019.

[40] Y. Povstenko, T. Kyrylych, Fractional thermoelasticity problem for an infinite solid with a penny-shaped crack under prescribed heat flux across its surfaces, Philosophical Transactions of the Royal Society A, 378, 20190289, 2020.

[41] W. Peng, Y. Ma, C. Li, T. He, Dynamic analysis to the fractional order thermoelastic diffusion problem of an infinite body with a spherical cavity and variable material properties, Journal of Thermal Stresses, 43, 38-54, 2020.

[42] H. H. Sherief, E. M. Hussein, The effect of fractional thermoelasticity on two dimensional problems in spherical regions under axisymmetric distributions, Journal of Thermal Stresses, 43, 440-455, 2020.

[43] U. Köbler, Universality in the temperature dependence of the heat capacity of magnetic solids, International Journal of Thermodynamics, 23, 147-174, 2020.

[44] S. Thakare, M. S. Warbhe, N. Lamba, Time fractional heat transfer analysis in nonhomogeneous thick hollow cylinder with internal heat generation and its thermal stresses, International Journal of Thermodynamics, 23, 281-302, 2020.

[45] B. Niezgoda-Żelasko, Analysis of entropy generation rate during non-adiabatic ice slurry flow in pipes, International Journal of Thermodynamics, 23, 25-32, 2020.

[46] M. T. Çoban, S. Kavas, Thermodynamic and thermophysical properties of dry air by using cubic peng-robinson eos for gas mixtures, International Journal of Thermodynamics, 23, 139-145, 2020.

[47] Y. Mohammadi, M. Rahmani, Temperature-dependent buckling analysis of functionally graded sandwich cylinders, Journal of Solid Mechanics, 12, 1-15, 2020. 
[48] M. Molla-Alipour, M. Shariyat, M. Shaban, Free vibration analysis of bidirectional functionally graded conical/cylindrical shells and annular plates on nonlinear elastic foundations, based on a unified differential transform analytical formulation, Journal of Solid Mechanics, 12, 385-400, 2020.

[49] M. Sarvandi, M. M. Najafizadeh, H, Seyyedhasani, Non-linear response of torsional buckling piezoelectric cylindrical shell reinforced with DWBNNTs under combination of electrothermo-mechanical loadings in elastic foundation, Journal of Solid Mechanics, 12, 505-520, 2020.

[50] M. Shekarzadeh, M. M. Najafizadeh, P. Yousefi, A. R. Nezamabadi, K. Khorshidi, Free vibration analysis of multi-layer rectangular plate with two magneto-rheological fluid layers and a flexible core, Journal of Solid Mechanics, 12, 847-861, 2020.

[51] S. P. Pawar, J. J. Bikram, G. D. Kedar, Thermoelastic behaviour in a multilayer composite hollow sphere with heat source, Journal of Solid Mechanics, 12, 883-901, 2020 .

[52] A. A. Kilbas, H. M. Srivastava, J. J. Trujillo, Theory and Applications of Fractional Differential Equations, Elsevier, Amsterdam 2006.

[53] K.B. Oldham, J. Spanier, The Fractional Calculus, Academic Press, New York, 1974.

[54] W. Nowacki, Thermoelasticity, Elsevier, 2013.

[55] N. Noda, R. B. Hetnarski, Y. Tanigawa, Thermal Stresses (Second Edition), Taylor and Francis, New York, 2003.

[56] M. N. Ozisik, Boundary Value Problems Of Heat Conduction, Dover Publications, New York, 1989.

\section{Appendix A}

Following Noda [4], we express the thermal conductivity $k(T)$ in terms of exponential law as

$k(T)=k_{0} \exp \left(\varpi_{1} T\right), \varpi_{1}<0$

Using equation (A1), equation (11) becomes

$\Theta=\left(k_{0} / \varpi_{1}\right)\left[\exp \left(\varpi_{1} T\right)-1\right]$

Using equation (A2) in equation (24), we obtain

$$
T(x, y, z, t)=\frac{1}{\varpi_{1}} \log _{e}[\Phi(x, y, z, t)+1]
$$

where

$$
\begin{aligned}
& \Phi(x, y, z, t)=\frac{\varpi_{1} P_{a} K i}{k_{0} \rho} \sum_{m=1}^{\infty}\left\{\left[\left[\overline{\bar{\Theta}}\left(\eta_{m}, \beta_{n}, z, t\right)\right]_{n=0} / b\right]\right. \\
& +(2 / b) \sum_{n=1}^{\infty}\left\{\left\{\left[\left[\xi_{1}(t)\right]_{l=0} / c\right]\right.\right. \\
& \left.+(2 / c) \times \sum_{l=1}^{\infty} \xi_{1}(t) \times \cos (l \pi z / c)\right\} \\
& \times \cos (n \pi y / b)\}\} \times\left\{\left(\left(\eta_{m} \cos \eta_{m} x+B i_{1} \sin \eta_{m} x\right)\right)\right. \\
& \left.\times\left(\sin \eta_{m} a-\left(B i_{1} / \eta_{m}\right) \cos \eta_{m} a+\left(B i_{1} / \eta_{m}\right)\right)\right\}
\end{aligned}
$$

We use the following logarithmic expansion $\log _{e}[\Phi(x, y, z, t)+1]=[\Phi(x, y, z, t)]$

$$
+(1 / 2)[\Phi(x, y, z, t)]^{2}
$$$$
+(1 / 3)[\Phi(x, y, z, t)]^{3}+\ldots \ldots \ldots .
$$

We observe that $[\Phi(x, y, z, t)]^{L}$ given in equation (A5) converges to zero as $L$ tends to infinity. Also the truncation error in equation (A5) is observed as $5.13 \times 10^{-5}$.

Hence, for the sake of brevity, neglecting the terms with order more than one, we obtain $\log _{e}[\Phi(x, y, z, t)+1] \cong \Phi(x, y, z, t)$

Hence equation (A3) becomes

$$
\begin{aligned}
& T(x, y, z, t) \cong \frac{P_{a} K i}{k_{0} \rho} \sum_{m=1}^{\infty}\left\{\left[\left[\overline{\bar{\Theta}}\left(\eta_{m}, \beta_{n}, z, t\right)\right]_{n=0} / b\right]\right. \\
& +(2 / b) \sum_{n=1}^{\infty}\left\{\left\{\left[\left[\xi_{1}(t)\right]_{l=0} / c\right]\right.\right. \\
& \left.\left.\left.+(2 / c) \times \sum_{l=1}^{\infty} \xi_{1}(t) \times \cos (l \pi z / c)\right\} \times \cos (n \pi y / b)\right\}\right\} \\
& \times\left\{\left(\left(\eta_{m} \cos \eta_{m} x+B i_{1} \sin \eta_{m} x\right)\right)\right. \\
& \left.\times\left(\sin \eta_{m} a-\left(B i_{1} / \eta_{m}\right) \cos \eta_{m} a+\left(B i_{1} / \eta_{m}\right)\right)\right\}
\end{aligned}
$$

